\title{
NUEVOS DATOS DE MOGOPLISTIDAE (INSECTA, ORTHOPTERA) Y PRIMER REGISTRO DE PARAMOGOPLISTES NOVAKI (KRAUSS, 1888) EN LA PENÍNSULA IBÉRICA
}

\author{
Pablo Barranco ${ }^{1, *}$ y Vicente M. Ortuño \\ ${ }^{1}$ Departamento de Biología y Geología, CITE-IIB, CECOUAL, Universidad de Almería, Ctra. Sacramento, s/n, 04120 La Cañada, Almería, España. \\ Email: pbvega@ual.es - ORCID iD: https://orcid.org/0000-0002-9411-3991 \\ ${ }^{2}$ Grupo de Investigación de Biología del Suelo y de los Ecosistemas Subterráneos. Departamento de Ciencias de la Vida, Facultad de Ciencias, \\ Universidad de Alcalá. E-28871 - Alcalá de Henares, Madrid, Spain. \\ Email: vicente.ortuno@uah.es - ORCID iD: https://orcid.org/0000-0001-5734-1621
}

*Autor para correspondencia: pbvega@ual.es

\section{RESUMEN}

Se estudian especímenes de Mogoplistidae colectados en las provincias de Alicante y Valencia, mediante la instalación de trampas de caída (pitfall) en la superficie del suelo, y con estaciones de muestreo subterráneo (EMS) emplazadas en el medio subterráneo superficial (MSS). Estas muestras se asignan a dos especies, Mogoplistes brunneus Serville, 1838, del que se colectaron cuatro hembras, y una especie aún no citada de la península ibérica, Paramogoplistes novaki (Krauss, 1888), de la que se recogieron 22 especímenes. El estudio morfológico y biométrico se realizó cotejando estos ejemplares con otra muestra de $P$. novaki proveniente de colectas en Croacia, y también con otras dos especies del género. La localización de ambas especies en las EMS, indica que éstas no sólo tienen hábitos epigeos, sino que también circulan por los intersticios del subsuelo de los canchales (depósitos coluviales).

Palabras clave: Paramogoplistes novaki, Mogoplistini, corología, autoecología, medio subterráneo superficial, Alicante, Valencia, España.

\section{ABSTRACT}

New data on Mogoplistidae (Insecta, Orthoptera) and the first record of Paramogoplistes novaki (Krauss, 1888) in the Iberian Peninsula

Several Mogoplistidae specimens collected in the provinces of Alicante and Valencia are studied by pitfall trapping, in the ground surface, and with underground sampling stations (EMS) located in the Mesovoid Shallow Substratum (MSS). These samples are assigned to two different species, Mogoplistes brunneus Serville, 1838, with four females collected, and a species not reported yet from the Iberian Peninsula, Paramogoplistes novaki (Krauss, 1888), 22 specimens of which were collected. The morphological and biometric study was carried out by comparing these specimens with another sample of $P$. novaki collected in Croatia, and also with other species of the genus. The location of both species in the EMS indicates that not only have these species epigeal habits, but they also circulate through the interstices of the subsoil of the scree (colluvial deposits).

Keywords: Paramogoplistes novaki, Mogoplistini, corology, autoecology, mesovoid shallow substratum, Alicante, Valencia, Spain.

Recibido/Received: 24/02/2020; Aceptado/Accepted: 29/07/2021; Publicado en línea/Published online: 05/10/2021

Cómo citar este artículo/Citation: Barranco, P. \& Ortuño, V. M. 2021. Nuevos datos de Mogoplistidae (Insecta, Orthoptera) y primer registro de Paramogoplistes novaki (Krauss, 1888) en la península ibérica. Graellsia, 77(2): e148. https://doi. org/10.3989/graellsia.2021.v77.309

Copyright: (c) 2021 SAM \& CSIC. Este es un artículo de acceso abierto distribuido bajo los términos de una licencia de uso y distribución Creative Commons Reconocimiento 4.0 Internacional (CC BY 4.0). 


\section{Introducción}

La familia Mogoplistidae está constituida por tres subfamilias (Cigliano et al., 2021), una de ellas fósil, de las cuales únicamente Mogoplistinae se halla en la península ibérica, y está representada por dos tribus y cuatro géneros. Gorochov (2020) reubica los géneros al subdividir la tribu Arachnocephalini Gorochov, 1984 en tres subtribus: 1) la subtribu Arachnocephalina Gorochov, 2020, con el género Arachnocephalus Costa, 1855 y una única especie $A$. vestitus Costa, 1855 ; 2) la subtribu Pseudomogoplistina, Gorochov, 2020 que aloja únicamente al género Paramogoplistes Gorochov, 1984, con P. squamiger (Fischer, 1853) y P. vicentae Gorochov, 1996; 3) la tribu Mogoplistini Brunner von Wattenwyl, 1873 con dos géneros, Mogoplistes Serville, 1838, con M. brunneus Serville, 1838 y Paramogoplistes Gorochov, 1984, con P. dentatus Gorochov \& Llorente, 2001 y P. ortini Llucià-Pomares, 2015.

Los grillos europeos de esta familia, denominados grillos con escamas, son de pequeño tamaño, inferior a un centímetro, y se caracterizan por tener un apterismo completo, o casi completo. Únicamente los machos de $M$. brunneus poseen tegminas reducidas, aunque no visibles, pues están cubiertas por el pronoto. Desde hace tiempo, muy diversos ejemplares de las especies ibéricas de Paramogoplistes han sido asignados a M. brunneus de forma errónea (Gorochov \& Llorente, 2001; Ferreira \& Grosso-Silva, 2008; Llucià-Pomares, 2015). Los requerimientos ecológicos de las especies de esta familia son muy diversos. Por ejemplo, las especies de los géneros Mogoplistes y Paramogoplistes se observan en el suelo, entre la hojarasca o debajo de piedras; las especies de Pseudomogoplistes en el litoral, concretamente en la zona de pleamar, y $A$. vestitus es la única especie que se localiza habitualmente sobre la vegetación.

En el norte de la provincia de Alicante, se han llevado a cabo muestreos sistematizados de fauna del medio subterráneo superficial (MSS), y epiedáfica, desde finales del año 2011 hasta finales de 2012, mediante la utilización de dispositivos pasivos de captura. Entre el material capturado se hallan algunos especímenes de Orthoptera pertenecientes a la familia Mogoplistidae.

\section{Material y métodos}

El área de estudio se circunscribe a los macizos montañosos y barrancos de la mitad septentrional de la provincia de Alicante, y a un enclave (barranco) del sur de la provincia de Valencia (Fig. 1A), relieves que forman parte del Sistema Penibético. Los materiales geológicos son de naturaleza calcárea depositados sobre margas que afloran en los fondos de valle (Estévez et al., 2004). Las temperaturas experimentan una notable amplitud térmica estacional, siendo las bajas temperaturas invernales promotoras de fuertes heladas (Padilla Blanco, 1995), precipitaciones en forma de nieve (Segura Martí, 1985, 1991), y veranos con temperaturas superiores a $\operatorname{los} 30^{\circ} \mathrm{C}$ (Gualda Gómez, 1988). En general la precipitación es escasa y torrencial, si bien la precipitación media de muchos de estos relieves ronda los 600-700 mm, aunque en algunas zonas se hallan entre los 240 y $380 \mathrm{~mm}$ (Padilla Blanco, 1995). Otros enclaves, con peculiar orografía y proximidad a la costa promueven el desarrollo de lluvias locales (Morales et al., 1983) y criptoprecipitación. Los cursos fluviales, a menudo encajonados, permanecen secos la mayor parte del año para tornarse, momentáneamente, caudalosos según el régimen de lluvias y las características propias del cauce (Vilà Valentí, 1961; Segura, 1990). La vegetación en gran parte de esta área geográfica está condicionada por un proceso de desertificación (Meléndez Pastor et al., 2009; Recatalá Boix et al., 2011), si bien buena parte de la zona montañosa ha servido de refugio climático para determinados vegetales que ahora, para esta área, se consideran relictos (Marco, 2001); tal es el caso de las especies eurosiberianas Taxus baccata L., Ilex aquifolium L, y Rhamnus alpinus L., o como Genista longipes Pau o Vella spinosa Boiss., endemismos béticos que, en estas montañas, alcanzan su límite de distribución septentrional.

Con el fin de poder cotejar los componentes faunísticos subterráneos y epiedáficos, se instalaron en nueve depósitos coluviales de enclaves montañosos (Fig. 1A), un total de 40 Estaciones de Muestreo Subterráneo (EMS), a las que se asociaron 40 trampas pitfall de superficie. De igual modo, se muestrearon los depósitos aluviales de 16 enclaves de cauces fluviales de aguas temporales (Fig. 1A), instalando 16 EMS y 16 trampas pitfall (ver Ortuño et al., 2013).

Las EMS están detalladamente descritas, e ilustradas, en otras publicaciones (Ortuño et al., 2013, 2020; entre otras). De un modo sucinto diremos que son cilindros de PVC de $11 \mathrm{~cm}$ de diámetro, y de $1 \mathrm{~m}$ de longitud (para depósitos coluviales y aluviales) o de $0,75 \mathrm{~m}$ y $0,50 \mathrm{~m}$ (para depósitos aluviales), y multiperforados (orificios de $8 \mathrm{~mm}$ de diámetro) en su mitad inferior que se entierran, verticalmente, a ras de suelo. En su interior, hasta tocar fondo, se instala una trampa pitfall con propilenglicol como conservante, y como cebo sólido, un vial con queso azul. Las tramas pitfall de superficie, se instalaban de forma convencional, a ras de suelo, y haciendo uso del mismo conservante que en las EMS, quedaban cubiertas con algunas piedras con el fin de protegerlas de las inclemencias meteorológicas y de la actividad de grandes animales. 

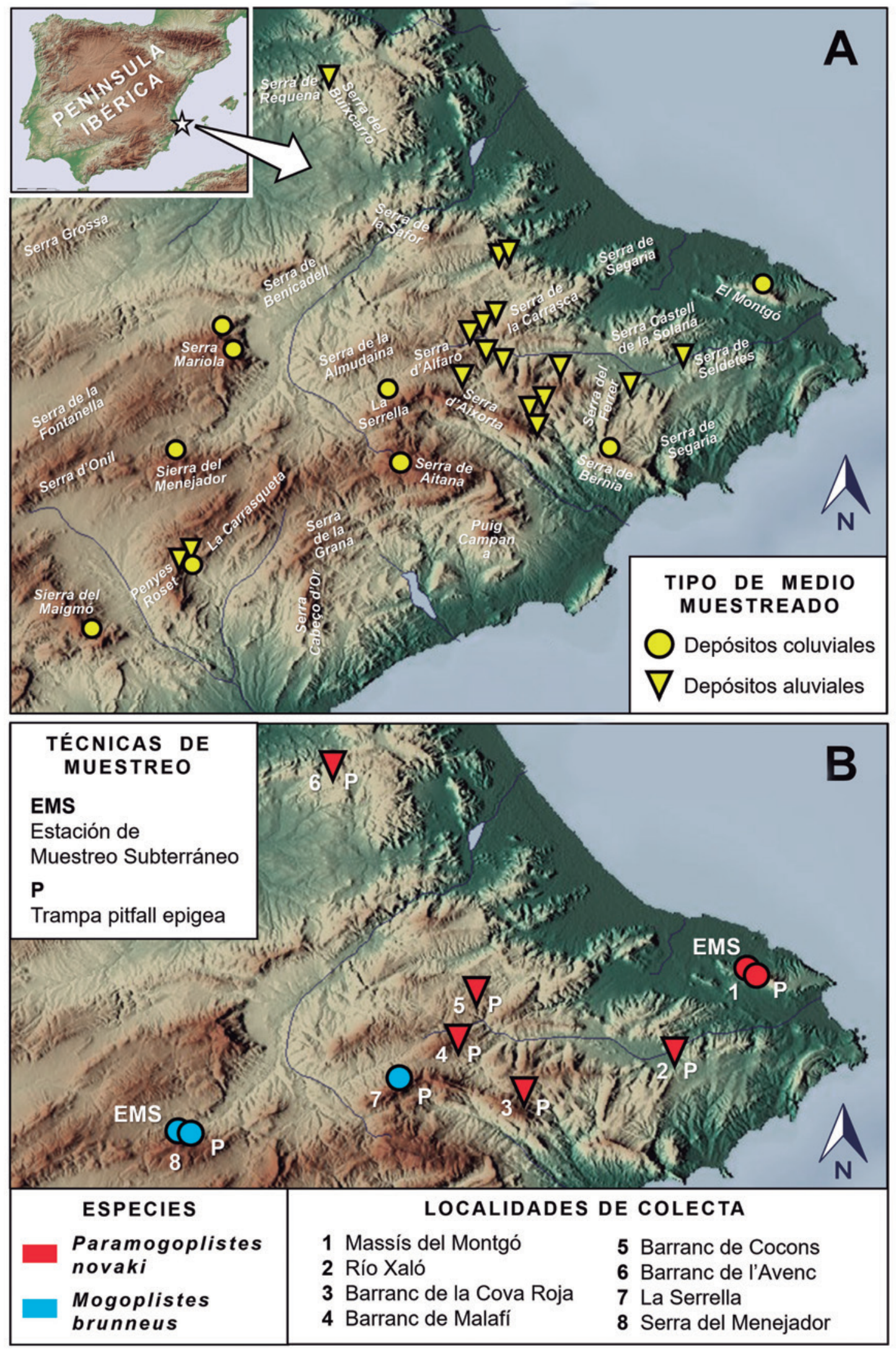

Fig. 1.- Área de muestreo (norte de Alicante y sur de Valencia): A) nombre de los relieves más notables y conjunto de enclaves, coluviales y aluviales, muestreados; B) se indican los enclaves en los que se ha colectado Paramogoplistes novaki y Mogoplistes brunneus, y se especifica el método de colecta.

Fig. 1.- Sampling area (north of Alicante and south of Valencia): A) name of the most notable reliefs and set of localities, colluvial and alluvial mesovoid shallow substratum sampled; B) localities in which Paramogoplistes novaki and Mogoplistes brunneus have been collected are indicated, and the collection method is specified. 


\section{Material estudiado}

Para el desarrollo de este trabajo, se han estudiado: 1) los ejemplares de Mogoplistidae capturados en las provincias de Alicante y Valencia (Fig. 1B); 2) grillos pertenecientes a otras capturas ibéricas del género Paramogoplistes; 3 ) ejemplares de $P$. novaki (Krauss, 1888) provenientes de colectas en Croacia. También se incluye el material de $M$. brunneus proveniente de Mallorca porque se trata de un espécimen adulto que confirma la presencia de esta especie en dicha isla. El material estudiado del MSS, se depositará en el Centro de Colecciones de la Universidad de Almería (CECOUAL), otro material de comparación en la colección P. Barranco y el prestado por otras instituciones europeas, se retornará a las mismas.

\section{Paramogoplistes novaki (Krauss, 1888)}

EsPaña: 1 macho (m) y 3 hembras (h), Massís del Montgó, 313 m s.n.m., 31SBD4900, Dènia (Alicante), 01-07-2012/31-102012, EMS; 1 ninfa (n), ídem, pitfall; 2 m, 2 h y 1 n, ídem, 322 m s.n.m., pitfall; 1 h, Barranc de Malafí, 478 m s.n.m., 30SYH4494, Castell de Castells (Alicante), 12-07-2012/2209-2012, pitfall; $1 \mathrm{~h}$, Barranc de Cocons, $407 \mathrm{~m}$ s.n.m., 30SYH4698, Vall D'Ebo (Alicante), 12-07-2012/22-09-2012; pitfall; $1 \mathrm{n}$, Barranc de la Cova Roja, 537 m s.n.m., 30SYH4988, Tárbena (Alicante), 25-04-2012/28-07-2012, pitfall; 7 n, Río Xaló, 103 m s.n.m., 31SBC4394, Gata de Gorgos (Alicante), 17-04-2012/10-07-2012, pitfall; $1 \mathrm{~h}$ y $1 \mathrm{n}$, Barranc de l'Avenc, 185 m s.n.m., 30SYJ2620, Pla de Corrals, Simat de Valldigna (Valencia), 12-07-2012/22-09-2012, pitfall [todos V.M. Ortuño et al. leg., CECOUAL coll.]. Croacia: $2 \mathrm{~m}$, Krčić River, Dinara Mt., 370 m s.n.m., (Knin), 20-10-2014, pitfall; 1 m, ídem, 2808-2014, pitfall; $1 \mathrm{~m}$, ídem, 24-09-2014, pitfall [todos Rebrina leg., Division of Zoology, Dep. Biology, Univ. Zagreb coll.].

Paramogoplistes dentatus Gorochov \& Llorente, 2001

Portugal: $2 \mathrm{~m}$ y 1 h, Cabeça, (Concelho de Seia), 22-06-1999, S. Ferreira leg. CINBIO coll.

\section{Paramogoplistes ortini Llucià-Pomares, 2015}

EsPaña: 1 h, ICE La Aduana (Córdoba), 05-11-1991; $1 \mathrm{~m}$, Sierra Horconera, Priego de Córdoba (Córdoba), 07-08-1992; $1 \mathrm{~m}$ y 1 h, Ermita Virgen de la Sierra, Cabra, (Córdoba), 09-09-1995; $1 \mathrm{~m}$ y 2 h, Cerro del Montecillo, 30-08-2001; 1 h, La Nava, Cabra, (Córdoba), 30-08-2019; 1h, Garganta del Capitán, 142 m s.n.m., 30STF7402, Los Barrios (Cádiz), 16-09-2019 [todos M. Baena leg., P. Barranco coll.].

\section{Mogoplistes brunneus Serville, 1839}

ESPAÑA: 1 h, TIV Monte Menut, 581 m s.n.m., 31SDE9109, Escorca (Mallorca, Islas Baleares), 04-07-2010, encinar, E. González leg., P. Barranco coll.; 1 h, La Serrella, 1000 m s.n.m., 30SYH3488, Quatretondeta (Alicante), 26-07-2012/3010-2012, pitfall; 2 h, Serra del Menejador, Parc Natural del Carrascar de la Font Roja, 1260 m s.n.m., 30SYH1381, Alcoi (Alicante), 12-07-2012/31-10-2012, EMS; 1 h, ídem, pitfall; [todos V.M. Ortuño et al. leg., CECOUAL coll.].

\section{Resultados}

Los Mogoplistidae capturados en la provincia de Alicante pertenecen a dos especies diferentes,
Mogoplistes brunneus, del que se colectaron cuatro hembras, y una especie aún no citada de la península ibérica, de la que se recogieron 22 especímenes. El estudio morfológico y biométrico de estos ejemplares permitió asignarlos a la especie Paramogoplistes novaki (Krauss, 1888), identificación que se ha corroborado al cotejarlos con otros individuos de esta especie, provenientes de Croacia, los cuales formaron parte de la serie que se utilizó en el estudio de Rebrina \& Brigić (2017). La comparación con otras especies de este género presentes en España también resultó enormemente clarificadora en pos de conocer su identidad específica. La forma de la placa subgenital en ambos sexos, la forma del oviscapto y la genitalia masculina son concordantes con los rasgos anatómicos descritos para $P$. novaki, y recogidos en la iconografía de Rebrina \& Brigić (2017).

Paramogoplistes novaki se diferencia de las otras especies del género en un conjunto de caracteres que, en lo sustancial, son los siguientes: a) la placa subgenital del macho es escotada en el centro del margen posterior, a diferencia de lo que manifiesta $P$. ortini y $P$. dentatus que muestran la placa redondeada, y también los paraproctos son mucho más grandes; b) el oviscapto es inerme, si bien en ello coincide con $P$. ortini; c) la genitalia masculina es mayoritariamente membranosa, con dos escleritos distales, asimétricos y de forma sinuosa que conforman un hueco en forma de bocallave. Paramogoplistes kinzelbachi (Harz, 1976) presenta una placa subgenital masculina diferente de la de otras especies mencionadas, pues es transversa y en el borde distal posee una evaginación redondeada; la genitalia es también mayoritariamente membranosa con escleritos simétricos y la abertura cruciforme.

En general, los ejemplares de $P$. dentatus son de mayor tamaño para todos los parámetros considerados, seguidos de $P$. novaki (aunque los especímenes de origen croata son sensiblemente más grandes que los ibéricos), P. ortini y, finalmente P. kinzelbachi, que son los más pequeños (Tabla 1). El pronoto de los ejemplares croatas de $P$. novaki es el más cuadrangular en ambos sexos. Por el contrario, los machos de esta especie, provenientes de Alicante, presentan el pronoto más transverso, con el valor más bajo de LP / AP.

Como indican Rebrina \& Brigić (2017), la correcta identificación de las hembras, tanto ninfas como adultas de $P$. novaki y $M$. brunneus presentan una distribución solapada en Croacia, pasa por reconocer la diferencia de tamaño (M. brunneus es más grande) y la forma de la placa subgenital. Todos los parámetros que se consideren son mayores para M. brunneus, y además, ésta presenta una escotadura en el ápice de la placa subgenital, mientras que $P$. novaki carece de escotadura, por lo que el ápice es recto, o ligeramente convexo. 
Tabla 1.- Valores biométricos de las especies del género Paramogoplistes. Abreviaturas: Ali: Alicante; AP: anchura del pronoto; ASg: anchura de la placa subgenital; CR: Croacia; GR: Grecia; LMf: longitud del metafémur; LMt: longitud de la metatibia; LO: longitud del oviscapto; LP: longitud del pronoto; LP/AP relación entre la anchura y longitud del pronoto; LSg: longitud de la placa sugenital; PO: Portugal; sPI: sur península ibérica. ( $\left.{ }^{*}\right)$ Datos obtenidos de la bibliografía: para P. novaki de Croacia (Rebrina \& Brigić, 2017), para P. ortini (Llucià-Pomares, 2015) y para P. kinzelbachi (Harz, 1976).

Table 1.- Biometric values of the species of the genus Paramogoplistes. Abbreviations: Ali: Alicante; AP: width of pronotum; ASg: width of the subgenital plate; CR: Croatia; GR: Greece; LMf: length of hind femur; LMt: length of the medium tibia; LO: length of the ovipositor; LP: pronotum length; LP/AP ratio between the width and length of the pronotum; LSg: length of the sugenital plate; PO: Portugal; sPI: southern Iberian Peninsula. ( $\left.{ }^{*}\right)$ Data obtained from the bibliography: for P. novaki from Croatia (Rebrina \& Brigić, 2017), for P. ortini (Llucià-Pomares, 2015) and P. kinzelbachi (Harz, 1976).

\begin{tabular}{|c|c|c|c|c|c|c|c|c|c|c|}
\hline & & & ASg & LSg & LO & AP & LP & LMf & LMt & LP / AP \\
\hline Paramogoplistes novaki & Ali & $f$ & $0,98 \pm 0,06$ & $0,90 \pm 0,06$ & $1,51 \pm 0,06$ & $1,82 \pm 0,09$ & $1,38 \pm 0,19$ & $3,48 \pm 0,25$ & $2,25 \pm 0,14$ & $0,76 \pm 0,08$ \\
\hline Paramogoplistes novaki & $\mathrm{CR}^{*}$ & $f$ & $1,07 \pm 0,09$ & $0,95 \pm 0,06$ & $1,53 \pm 0,15$ & $1,69 \pm 0,09$ & $1,46 \pm 0,07$ & & & $0,86 \pm 0,05$ \\
\hline Paramogoplistes ortini & $\left.\mathrm{sP}\right|^{*}$ & $f$ & & & $1,50 \pm 0,23$ & $1,81 \pm 0,13$ & $1,32 \pm 0,14$ & $3,47 \pm 0,27$ & $2,29 \pm 0,18$ & $0,73 \pm 0,13$ \\
\hline Paramogoplistes dentatus & $\mathrm{PO}$ & $f$ & 1,18 & 1,08 & $1,78 \pm 0,23$ & 2,25 & $1,76 \pm 0,04$ & $4,05 \pm 0,03$ & $2,73 \pm 0,03$ & $0,78 \pm 0,14$ \\
\hline Paramogoplistes kinzelbachi & $\mathrm{GR}^{*}$ & $f$ & & & $1,5-1,8$ & & $1,3-1,4$ & $3-3,5$ & & \\
\hline Paramogoplistes novaki & Ali & $\mathrm{m}$ & $1,27 \pm 0,15$ & $0,59 \pm 0,08$ & & $1,64 \pm 0,08$ & $1,16 \pm 0,09$ & $3,23 \pm 0,18$ & $2,08 \pm 0,1$ & $0,71 \pm 0,08$ \\
\hline Paramogoplistes novaki & $\mathrm{CR}^{*}$ & $\mathrm{~m}$ & $1,38 \pm 0,07$ & $0,45 \pm 0,05$ & & $1,56 \pm 0,08$ & $1,33 \pm 0,08$ & & & $0,85 \pm 0,03$ \\
\hline Paramogoplistes ortini & $\left.\mathrm{sP}\right|^{*}$ & $\mathrm{~m}$ & & & & $1,48 \pm 0,15$ & $1,26 \pm 0,15$ & $2,91 \pm 0,33$ & $2,01 \pm 0,03$ & $0,76 \pm 0,01$ \\
\hline Paramogoplistes dentatus & $\mathrm{PO}$ & $\mathrm{m}$ & $1,35 \pm 0,09$ & $0,33 \pm 0,03$ & & $1,79 \pm 0,01$ & $1,48 \pm 0,18$ & $3,52 \pm 0,18$ & $2,35 \pm 0,15$ & $0,77 \pm 0,00$ \\
\hline Paramogoplistes kinzelbachi & $\mathrm{GR}^{*}$ & $\mathrm{~m}$ & & & & & $1,1-1,2$ & $2,7-3$ & & \\
\hline
\end{tabular}

\section{Discusión}

Krauss (1888) describe Mogoplistes novaki de la isla croata de Hvar (también conocida por su nombre griego, Lesina), y lo hace a partir de una única hembra, diferenciándola de $M$. brunneus por la forma cuadrangular del pronoto con su margen posterior recto, y la placa subgenital densamente pilosa. Chopard (1927) describe Mogoplistes minuttissimus Chopard, 1927 del Monte Parnaso (Grecia), y para ello cuenta con un espécimen adulto de cada sexo. Posteriormente, el mismo Chopard (1968) sinonimiza esta especie con la especie que propuso Krauss. A su vez, Gorochov (1984) propone el género Paramogoplistes y designa $M$. novaki (a partir de ese momento P. novaki) como especie tipo, ilustrando entre otros caracteres, la genitalia masculina; es pertinente indicar que, en ese momento, Gorochov (1984) no incluye en Paramogoplistes a Mogoplistes kinzelbachi, especie descrita de la isla Kárpatos (Grecia). Años más tarde, esta especie áptera sí será considerada dentro de este género (Gorochov \& Llorente, 2001; Llucià-Pomares, 2015; Rebrina \& Brigić, 2017), aunque no está recogido en Cigliano et al. (2021).

Si bien $P$. novaki fue descrita de la isla Hvar (Krauss, 1888) en Croacia, no será hasta el siglo XXI cuando se colecte en Croacia continental (Rebrina \& Brigić, 2017; Skejo et al., 2018). A su presencia en Grecia, con los datos aportados por Chopard (1927), también hay que sumar el de Cerdeña (Ingrisch, 1983) y, más recientemente, el de la región de Lazio en Italia continental (Schmidt, 1996). Bien es verdad que las citas de Italia se consideran dudosas por un posible error en la identificación (Massa et al., 2013; Hochkirch et al. 2016). No obstante, el hallazgo de las poblaciones de Alicante, este de España, podría dar cierta verosimilitud a las citas italianas $\mathrm{y}$, sumado al conjunto de registros que se tienen de esta especie, perfilar un tipo de distribución que se hallaría entre dos corotipos, el Tirrénico, más restringido, y el Turánico-europeo, más amplio (ver Vigna Taglianti et al., 1992). La crisis de salinidad del Messiniense (Soria, 2007) explicaría este tipo de distribuciones que, de no mediar eventos de antropocoria, responden a antiguas conexiones terrestres de la cuenca mediterránea. Otras muchas especies de Artrópodos muestran distribuciones similares: los coleópteros Carabus (Macrothorax) morbillosus Fabricius, 1792 Cardiomera genei Bassi, 1834, o la línea de los Microtyphlus (sensu lato) y en particular el género Hypotyphlus Jeannel, 1937 (ver: Jeannel, 1937, 1942; Magistretti, 1965; Casale et al., 1989), las arañas del género Kaemis DeelemanReinhold, 1993 (ver Ferrández, 2018), o los milpiés del binomio Ommatoiulus ilicis (Brölemann, 1897) y Ommatoiulus corsicus (Brölemann, 1903) (ver Gilgado et al., 2015), por poner unos ejemplos.

La localización de $P$. novaki en la península ibérica constituye la primera cita para este territorio. Podría pertenecer a esta especie el ejemplar inmaduro capturado por Boscà en Valencia y reseñado por Bolívar (1878), del que señala no ajustarse a ninguna de las especies conocidas. La captura en cinco localidades de Alicante y una de Valencia, evidencia una amplia distribución en esta zona de España (Fig. 1B). Además, es destacable la captura de varios especímenes en una trampa (EMS) instalada en el medio subterráneo superficial (MSS), lo que indica que esta especie no sólo tiene hábitos epigeos, sino 
que también puede desenvolverse por los intersticios del subsuelo de los canchales (depósitos coluviales). Si bien, no debe ser un comportamiento muy asentado en esta especie, pues sólo fue detectada en el MSS de uno de los nueve enclaves de montaña muestreados, y en ningún de los 17 MSS aluviales muestreados; el resto de las capturas se corresponden con ambientes epigeos, si bien mayoritariamente aluviales (Fig. 1B). En este sentido, también es interesante el hábito dual detectado para $M$. brunneus, que se ha capturado igualmente tanto en trampas epigeas (P) como en trampas subterráneas (EMS), pero sin mostrar solapamiento con $P$. novaki, y circunscrita a depósitos coluviales en enclaves de montaña (Fig. 1B). Su captura también supone la primera cita para la provincia de Alicante.

Los diferentes estudios que se están desarrollando en el MSS, están poniendo de manifiesto la abundancia de grillos en estos hábitats, muy especialmente de los géneros Petaloptila Pantel, 1890 y Gryllomorpha Fieber, 1853, e incluso con una especie aparentemente exclusiva de este medio como es Nemobius interstitialis Barranco, Gilgado \& Ortuño, 2013. En el MSS de la Sierra de María (Almería) se ha capturado un individuo de Paramogoplistes, si bien no ha sido posible su identificación específica al tratarse de un ejemplar inmaduro. Es de esperar que nuevas prospecciones en estos hábitats subterráneos deparen más hallazgos de grillos de hábitos geófilos, aumentando así el conocimiento sobre la biodiversidad de Orthoptera en el ámbito ibérico.

\section{Agradecimientos}

Investigación financiada por el proyecto "Estudio del Medio Subterráneo Superficial en el Levante Español: hábitat de fauna relicta y de especial relevancia para la conservación de especies estenoica epiedáficas", con cargo al presupuesto CGL2010-19924 del Ministerio de España de Ciencia e Innovación; y por el programa I3 de "Incentivación de la Incorporación e Intensificación de la Actividad Investigadora" del Ministerio de Educación y Ciencia de España del que V.M. Ortuño fue el beneficiario. Hacemos constar nuestro agradecimiento a José Domingo Gilgado, Alberto JiménezValverde, Gonzalo Pérez-Suárez, Alberto Sendra y Juan J. HerreroBorgoñón, miembros del equipo de investigación que colaboraron activamente en el diseño y muestreo del proyecto desarrollado en la provincia de Alicante. También queremos mostrar nuestra gratitud a todos los colectores que nos han cedido material ibérico de Mogoplistinae para su estudio, así como a Fran Rebrina, Andreja Brigić y Zlatko Mihaljević, del Departamento de Zoología de la Universidad de Zagreb, por el préstamo de ejemplares croatas de Paramogoplistes novaki.

\section{Referencias}

Bolívar, I., 1878. Sinopsis de los ortópteros de España y Portugal. $4^{\mathrm{a}}$ parte. Anales de la Sociedad Española de Historia Natural, 7: 63-129.
Casale,A., Bastianini, M. \& Minniti, M. 1989. Sulla presenza in Toscana di Carabus (Macrothorax) morbillosus Fabricius (Coleoptera, Carabidae, Carabini) e sul suo significato zoogeografico. Frustula entomológica, N.S., 10(1987): 67-72.

Chopard, L., 1927. Descriptions de Gryllides nouveaux. Annales de la Société Entomologique de France, 96: 147-174.

Chopard, L., 1968. Fam. Gryllidae: Subfam. Mogoplistinae, Myrmecophilinae, Scleropterinae, Cachoplistinae, Pteroplistinae, Pentacentrinae, Phalangopsinae, Trigonidiinae, Eneopterinae; Fam. Oecanthidae, Gryllotalpidae. In: Beier, M. (ed.). Orthopterorum Catalogus. 12. Uitgeverij, W. Junk N. V.'s Gravenhage: 213-500.

Cigliano, M.M., Braun, H., Eades, D.C. \& Otte. D., 2021. Orthoptera Species File. Version 5.0/5.0. Disponible en http://Orthoptera.SpeciesFile.org [consultado 12 Aug. 2021].

Estévez, A., Vera, J.A., Alfaro, P., Andreu, J.M., TentManclús, J.E. \& Yébenes, A., 2004. Geología de la provincia de Alicante. Enseñanza de las Ciencias de la Tierra, 12(1): 2-15.

Ferrández, M.A. 2018. Sobre el estatus genérico de Harpactea aeruginosa Barrientos, Espuny \& Ascaso, 1994 (Araneae: Dysderidae). Revista Ibérica de Aracnología, 32: 137-138.

Ferreira, S. \& Grosso-Silva, J.M., 2008. On the true identity of the portuguese specimens of Mogoplistes brunneus Serville, 18439 (Orthoptera, Mogoplistidae). Boletín de la Sociedad Entomológica Aragonesa, 43: 325-326.

Gilgado, J.D., Enghoff, H., Tinaut, A., Mauriès, J.-P. \& Ortuño, V.M. 2015. Sierra Nevada (Granada, Spain): a high-altitude biogeographical crossroads for millipedes (Diplopoda), with first data on its MSS fauna and description of a new species of the genus Ceratosphys Ribaut, 1920 (Chordeumatida: Opisthocheiridae). Zootaxa, 4044(3): 391-410. https://doi.org/10.11646/ zootaxa.4044.3.4

Gorochov, A.V., 1984. A contribution to the taxonomy of modern Grylloidea (Orthoptera) with a description of new taxa. Zoologischeskij Zhurnal, 63(1): 1641-1651. [En ruso.]

Gorochov, A.V., 2020. New subtribes of Arachnocephalini (Orthoptera: Mogoplistidae) and a new genus and species of this tribe from South Africa. Zoosystematica Rossica. 29 (2): 347-352.

Gorochov, A.V. \& Llorente, V., 2001. Estudio taxonómico preliminar de los Grylloidea de España (Insecta, Orthoptera). Graellsia, 57(2): 95-139. https://doi. org/10.3989/graellsia.2001.v57.i2.281

Gualda Gómez, C.E., 1988. La Sierra de Mariola, aspectos geomorfológicos y biogeográficos. Secretariado de Publicaciones. Universidad de Alicante. Alicante.

Harz, K, 1976. Orthopteologische Beiträge XV. Nachrichtenblatt der Bayerischen Entomologen, 25: 54-58.

Hochkirch, A., Nieto, A., García Criado, M., Cálix, M., Braud, Y., Buzzetti, F.M., Chobanov, D., Odé, B., Presa Asensio, J.J., Willemse, L., Zuna-Kratky, T., Barranco Vega, P., Bushell, M.,Clemente, M.E., Correas, J.R., Dusoulier, 
F., Ferreira, S., Fontana, P., García, M.D., Heller, K-G., Iorgu I.Ș., Ivković, S., Kati, V., Kleukers, R., Krištín, A., Lemonnier-Darcemont, M.,Lemos, P., Massa, B., Monnerat, C., Papapavlou, K.P., Prunier, F., Pushkar, T., Roesti, C.,Rutschmann, F., Şirin, D., Skejo, J., Szövényi, G., Tzirkalli, E., Vedenina, V., Barat Domenech, J., Barros, F., Cordero Tapia, P.J., Defaut, B., Fartmann, T., Gomboc, S., Gutiérrez-Rodríguez, J., Holuša, J., Illich, I., Karjalainen, S., Kočárek, P., Korsunovskaya, O., Liana, A., López, H., Morin, D., Olmo-Vidal, J.M., Puskás, G., Savitsky, V., Stalling, T. \& Tumbrinck, J., 2016. European Red List of Grasshoppers, Crickets and Bushcrickets. Publications Office of the European Union. Luxembourg. 94 pp. Disponible en https://www.iucn. org/content/european-red-list-grasshoppers-crickets-andbush-crickets [consultado 12 Aug. 2021].

Ingricsh, S., 1983. Neue Arten und faunistisch bemerkenswerte Nachweise von Orthopteren auf Sardinien. Nachrichtenblatt der Bayerischen Entomologen, 32(3): 88-94.

Jeannel, R. 1937. Les Bembidiides endogés (Col. Carabidae) Monographie d'une lignée gondwanienne. Revue française d'entomologie, 3(4): 241-396.

Jeannel, R. 1942. Coléoptères Carabiques (2e partie). Faune de France, 40: 572-1 173. Lechevalier, Paris.

Krauss, H.A., 1888. Orthoptera duo nova ex insula Lesina Dalmatiae. Wiener Entomologische Zeitung, 7: 117118.

Llucià-Pomares, D., 2015. Descripción de una nueva especie del género Paramogoplistes Gorochov, 1984, del sur de la Península Ibérica (Orthoptera, Mogoplistidae). Boletín de la Sociedad Entomológica Aragonesa, 56: 39-48.

Magistretti, M. 1965. Fauna d'Italia, 8. Coleoptera, Cicindelidae, Carabidae. Calderini. Bolonia. 512 pp.

Marco, J.A., 2001. Aitana como espacio singular. Publicaciones de la Universidad de Alicante. Alicante.

Massa, B., Fontana, P., Buzzetti, F.M., Kleukers, R.M.J.C. \& Odé, B., 2013. Orthoptera. 48. Fauna d'Italia. Calderini. Milano. 563 pp.

Meléndez Pastor, I., Navarro Pedreño, J., Gómez Lucas, I. \& Koch, M., 2009. Análisis de series temporales de vegetación obtenidas mediante teledetección como herramienta para el seguimiento de procesos de desertificación. In: Romero Díaz, M.A., Belmonte Serrato, F., Alonso Sarria, F. \& López Bermúdez F. (eds.). Avances en estudios sobre desertificación: aportaciones al Congreso Internacional sobre Desertificación en memoria del profesor John B. Thornes. Universidad de Murcia. Murcia: 339-342.

Morales Gil, A., Bru Ronda, C. \& Box Amorós, M., 1983. Morfología en la umbría de la Sierra de Bernia. Investigaciones Geográficas, 1: 117-145.

Ortuño, V.M., Barranco, P., Jiménez-Valverde, A. \& Sendra, A., 2020. El relicto glacial Leistus (Pogonophorus) puncticeps Fairmaire \& Laboulbène, 1854 (Coleoptera, Carabidae): nuevos datos sobre distribución, autoecología y presencia en el Medio Subterráneo Superficial (MSS). Graellsia, 76(1): e107. https://doi.org/10.3989/ graellsia.2020.v76.255

Ortuño, V.M., Gilgado, J.D., Jiménez-Valverde, A., Sendra, A., Pérez-Suárez, G. \& Herrero-Borgoñón, J.J., 2013. The "Alluvial Mesovoid Shallow Substratum", a New Subterranean Habitat. PLoS ONE, 8(10): e76311. https://doi.org/10.1371/journal.pone.0076311

Padilla Blanco, A., 1995. El poblamiento vegetal en las sierras de Peñarroya y Cuartel: aspectos evolutivos y situación actual. Instituto Universitario de Geografía. Universidad de Alicante. Alicante.

Rebrina, F. \& Brigić, A., 2017. Morphometric variability and life history traits of the rare Paramogoplistes novaki in comparison to Mogoplistes brunneus (Orthoptera: Mogoplistidae). Annales de la Société Entomologique de France, New Series, 53(5): 313-323. https://doi.org/ 10.1080/00379271.2017.1344565

Recatalá Boix, L., Añó Vidal, C., Valera Lozano, A. \& Sánchez Díaz, J., 2011. Control de la degradación y uso sostenible del suelo. Libro de Actas del V Simposio Nacional sobre Control de la Degradación y Uso Sostenible del Suelo, Murcia: 87-90.

Schmidt, G.H., 1996. Biotopmässige Verteilung und Vergesellschaftung der Saltatoria (Orthoptera) im Parco Nazionale del Circeo, Lazio, Italien. Deutsche Entomologische Zeitschrift, 43(1): 9-75. https://doi. org/10.1002/mmnd.19960430104

Segura, F.S., 1990. Las ramblas valencianas. Algunos aspectos de hidrología, geomorfología y sedimentología. Tesis doctoral. Universitat de València. Valencia.

Segura Martí, J.M. 1985. La industria de la nieve en las montañas alicantinas. Narria: Estudios de artes y costumbres populares, 37-38: 2-11.

Segura Martí, J.M., 1991. Los pozos de nieve. In: Oliver Narbona, M. (coord.), Historia de la provincia de Alicante, vol. 7. Mediterráneo Ediciones, Murcia: 313340.

Skejo, J., Rebrina, F., Szövényi, G., Puskás, G. \& Tvrtković, N., 2018. The first annotated checklist of Croatian crickets and grasshoppers (Orthoptera: Ensifera, Caelifera). Zootaxa, 4533(1): 1-95. https:// doi.org/10.11646/zootaxa.4533.1.1

Soria, J.M. 2007. La crisis de salinidad del Messiniense. Enseñanza de las Ciencias de la Tierra, 15(1) 47-55.

Vigna Taglianti. A., Audisio, P.A., Belfiore, C., Biondi, M., Bologna, M.A., Carpaneto, G.M., de Biase, A., de Felici, S., Piattella, E., Racheli, T., Zapparoli, M. \& Zoia, S. 1992. Riflessioni di gruppo sui corotipi fondamentali della fauna W-paleartica ed in particolare italiana. Biogeographia - The Journal of Integrative Biogeography, 16: 159-179. https://doi.org/10.21426/ b616110375

Vilà Valentí, J., 1961. L'irrigation par nappes pluvials dans la Sud-Est espagnol. Méditerranée, 2: 18-32. 